\title{
On some problems of synthesis of spatial five-bar hinged mechanisms with two degrees of freedom
}

\author{
Nodar Davitashvili", Otar Gelashvili \\ Department of Transport and Mechanical Engineering of Georgian Technical University, Tbilisi, Georgia \\ Email address: \\ nodav@pam.edu.ge (N. Davitashvili), gelashviliotari@mail.ru (O. Gelashvili)
}

\section{To cite this article:}

Nodar Davitashvili, Otar Gelashvili. On Some Problems of Synthesis of Spatial Five-Bar Hinged Mechanisms with Two Degrees of Freedom. International Journal of Mechanical Engineering and Applications. Vol. 2, No. 6, 2014, pp. 104-110. doi: 10.11648/j.ijmea.20140206.14

\begin{abstract}
Solution of the problems of synthesis of spatial five-bar hinged mechanisms with two degrees of freedom task considering the angle of transmission and definition of conditions of existence of cranks is given in the paper. A possibility of movement of the designed mechanism without seizure that is depended on shape and sizes of the mechanism links is envisaged. At solution of the problem of synthesis first are determined sizes of the mechanism couples considering the angle of transmission and then are determined the limits of variation of the angle of transmission depending on sizes of the mechanism links. The conditions are ascertained which must be met by sizes of the spatial five-bar mechanism links so that the two links adjoining to the frame are crank (theorem on existence of cranks in the spatial five-bar mechanism).
\end{abstract}

Keywords: Synthesis, Angle of Transmission, Spatial Five-Bar Mechanism, Coupler, Conditions of Existence of Crank

\section{Introduction}

Spatial mechanism with two degrees of freedom rank high among numerous hinged mechanisms used in practice, which in contrast to four-bar mechanisms, can realize more complex laws of motion favouring at the same time rise of the machines efficiency and optimization of the technological processes.

A distinctive feature of these mechanisms is their capacity for variation of the coupler point locus from the simple to the more complex form in the course of operation at variation of angular velocities of the input links considering directions of motions and their mutual initial inter-location at constant lengths of the links.

These circumstances are directly related with selection of length of the output links of mechanisms and predetermine necessary conditions for creation of compact, lightweight mechanisms, especially in the fields of space and robotics industry, decreasing at this consumption of materials and energy.

The spatial five-bar hinged mechanisms with two degrees of freedom are widely used in various devices, robotics systems as well as in industrial practice as operating members [1, 2] and so on.

The researches into hinged mechanisms with two degrees of freedom started at the end of XIX and beginning of XX centuries by first studying planar five-bar mechanisms with two input links.

Solution of the problem on locations for the spatial mechanisms with several degrees of freedom and spherical five-bar mechanisms with two degrees of freedom was first considered by A.G. Ovakimov [3] only in the 70-ies of the XX century.

The problems of kinematical analysis of the spatial five-bar mechanism of type RCRCR with one degree of freedom were considered in the work of J. Duffi and G.I. Habib-Olahi. [4]. The analytical formulae were derived with the use of spherical trigonometry. The positions of output links of the mechanism are determined by the forth order equation and in the work of J. Duffi [5] for determination of displacements of the spatial five-bar mechanism were used the dual equations.

Much attention was given to the problems of analysis, synthesis, kinetostatics and dynamics of mechanisms with two degrees of freedom by Georgian scientists having carried out deep analysis of the mentioned mechanisms.

In should be noted in this aspect the works $[6,7]$ of N.S. Davitashvili. In the work [6] is given analysis of influence of parameters of the five-bar hinged mechanism on trajectories of the couplers points and in the work [7] - are studied problems of kinematics and precision of these mechanisms.

Besides the mentioned works numerous researches into analysis, synthesis, dynamics and precision of hinged 
mechanisms with two degrees of freedom are carried out by first author of these articles $[8,9,10]$. In the work [11] of N.S. Davitashvili and O. Gelashvili is given synthesis of the five-bar spatial mechanism with two degrees of freedom according to the trajectory.

In the presented work is carried out synthesis of the spatial five-bar hinged mechanism with two degrees of freedom taking into account the angle of transmission and conditions of existence of two cranks.

\section{Synthesis of the Spatial Five-Bar Mechanism Taking into Account the Angle of Transmission}

At solution of the problem of synthesis of spatial five-bar mechanisms with two degrees of freedom it is necessary to consider the following requirements: correct structure of the mechanism to be designed, kinematical precision of the movement to be performed, possibility of realization of the given movement by mechanism to be designed from the standpoint of dynamics, possibility of movement of the mechanism under action of applied forces with maximum possible efficiency (taking into account the angle of transmission to avoid their seizure) and finally, ensuring existence of cranks and reproduction of the given movement by the sizes of links of the synthesized mechanism.

\subsection{Dimensioning of Couplers of the Five-Bar Spatial Mechanism Taking into Account the Given Value of the Angle of Transmission}

Let's consider spatial five-link hinged leverage RSSRR type mechanism ABCDE (Fig. 1) with two degrees of freedom and determine the dimensions $\mathrm{BC}=\ell_{3}$ and $\mathrm{CD}=\ell_{4}$ of couplers with taking into account the given values of angle of transmission $\gamma$ $\left(\gamma_{\min }, \gamma_{\max }\right)$.

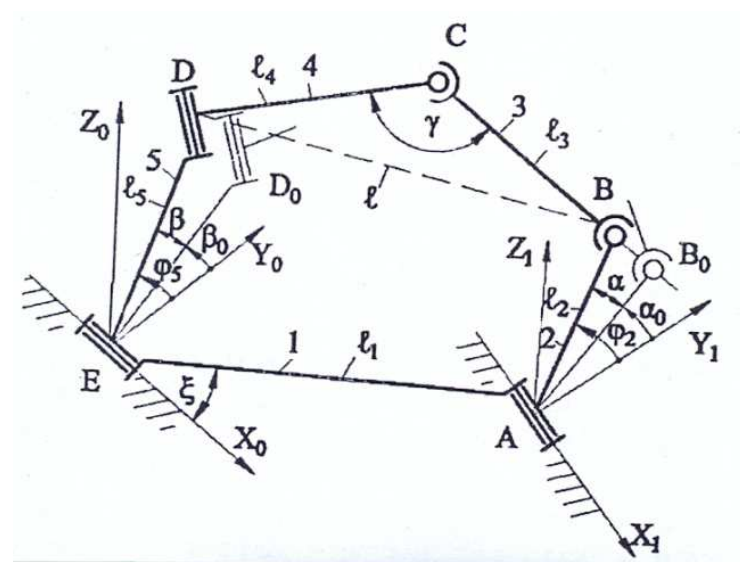

Figure 1. Spatial five-bar mechanism with two degrees of freedom.

For solution of this problem are given: dimensions of links $1,2,5$, designated as $\ell_{1}, \ell_{2}$, and $\ell_{5}$, angles $\alpha_{0}$ and $\beta_{0}$, specifying the initial position of the input $\mathrm{AB}_{0}$ and $\mathrm{ED}_{0}$ links 2 and 5, with respect to the frame 1 .

The laws of motion of the input links 2 and 5 are given as:

$$
\varphi_{2}=\varphi_{2}(t) \text { and } \varphi_{5}=\varphi_{5}(t)
$$

where

$$
\varphi_{2}=\alpha_{0}+\alpha(t) \text { and } \varphi_{5}=\beta_{0}+\beta(t) .
$$

Here $\alpha$ and $\beta-$ are the variable angles characterizing the motion of input links.

The gear ratios from link 5 to link 2 is defined as

$$
u_{52}=\frac{\varphi_{5}}{\varphi_{2}}=\frac{\omega_{5}}{\omega_{2}}=\frac{\beta}{\alpha}= \pm \frac{p}{q},
$$

where the sign $(+)$ is taken for the case when input links 2 and 5 are rotating in the same direction, and the sign (-) when they are rotating in the opposite directions..

It should be noted that by giving functions of displacements of the input links, gear ratio is also

$$
u_{52}=u_{52}(t) .
$$

We select two fixed coordinate systems for the input links 2 and 5 so that point $A$ is located in the plane $\mathrm{X}_{0} \mathrm{EY}_{0}$. The system $E X_{0} Y_{0} Z_{0}$ is selected for link 5, and system $A X_{1} Y_{1} Z_{1}$ for link 2.

Accordingly are given the coordinates of point $A\left(x_{0} y_{0} z_{0}\right)$ in the system $E X_{0} Y_{0} Z_{0}$ considering angle $\xi$ between axis $E X_{0}$ and frame 1:

$$
x_{0}=l_{1} \cos \xi ; \quad y_{0}=l_{1} \sin \xi ; \quad z_{0}=0 .
$$

The transformation of system $A X_{1} Y_{1} Z_{1}$ in the system $E X_{0} Y_{0} Z_{0}$ is carried out due the matrix:

$$
\left|\begin{array}{lll}
b_{11} & b_{21} & b_{31} \\
b_{12} & b_{22} & b_{32}=0 \\
b_{31} & b_{32} & b_{33}
\end{array}\right|
$$

where $b_{11}, b_{12}, \ldots, b_{33}$ - are directing cosines of axes of the system $A X_{1} Y_{1} Z_{1}$ with respect to the $E X_{0} Y_{0} Z_{0}$ system.

It is possible at first to set the four values of directing cosines, for example, $b_{11}, b_{12}, b_{31}$ and $b_{32}=0$, then for $b_{33}, b_{12}$, $b_{23}, b_{12}$ we will have:

$$
\begin{aligned}
& b_{33}=\sqrt{1-b_{31}^{2}} ; \quad b_{13}=-\frac{b_{11} b_{31}}{b_{33}} ; \\
& b_{23}=-\frac{b_{21} b_{31}}{b_{33}} ; \quad b_{12}=\sqrt{1-b_{11}^{2}-b_{31}^{2}} .
\end{aligned}
$$

The variable distance $\ell$ between movable joints $\mathrm{B}$ and $\mathrm{D}$, the value of that is depended on dimensions of frame $\ell_{1}$ and input links $2\left(\ell_{2}\right)$ and $5\left(\ell_{5}\right)$, initial position of input links $\left(\alpha_{0}, \beta_{0}\right)$, gear ratio $\left(u_{52}\right)$ between input links with taking into account directions of their movement can be determined in the system $E X_{0} Y_{0} Z_{0}$ with the help of formula for the distance between two points in space:

$$
\ell^{2}=\left(x_{B}-x_{D}\right)^{2}+\left(y_{B}-y_{D}\right)^{2}+\left(z_{B}-z_{D}\right)^{2},
$$

where 


$$
\begin{array}{ll}
x_{B}=0 ; & y_{B}=\ell_{2} \cos \varphi_{2} ; \quad z_{B}=\ell_{2} \sin \varphi_{2} . \\
x_{D}=0 ; & y_{D}=\ell_{5} \cos \varphi_{5} ; \quad z_{D}=\ell_{5} \sin \varphi_{5} .
\end{array}
$$

Considering the location of point $\mathrm{A}$ at the origin of the system, , the coordinates of point B CAN be written in the system $E X_{0} Y_{0} Z_{0}$ using matrix (6);

$$
\begin{aligned}
& x_{B}=b_{12} \ell_{2} \cos \varphi_{2}+b_{13} \ell_{2} \sin \varphi_{2}+\ell_{1} \cos \zeta \\
& y_{B}=b_{22} \ell_{2} \cos \varphi_{2}+b_{23} \ell_{2} \sin \varphi_{2}+\ell_{1} \sin \zeta \\
& z_{B}=b_{33} \ell_{2} \sin \varphi_{2} .
\end{aligned}
$$

Considering values (2), (10) and (11) expression (8) will take a form:

$$
\begin{aligned}
\ell= & \left\{\ell_{1}^{2}+\ell_{2}^{2}+\ell_{5}^{2}+2 \ell_{1} \ell_{2}\left[\cos \left(\alpha_{0}+\alpha\right) \cdot\right.\right. \\
& \cdot\left(b_{12} \cos \xi+b_{12} \sin \xi\right)+\sin \left(\alpha_{0}+\alpha\right) \cdot \\
& \left.\cdot\left(b_{31} \cos \xi+b_{23} \sin \xi\right)\right]- \\
& -2 \ell_{1} \ell_{5} \sin \xi \cos \left(\beta_{0}+u_{52} \alpha\right)- \\
& -2 \ell_{2} \ell_{5}\left[b_{22} \cos \left(\alpha_{0}+\alpha\right) \cos \left(\beta_{0}+u_{52} \alpha\right)+\right. \\
& +b_{23} \sin \left(\alpha_{0}+\alpha\right) \cos \left(\beta_{0}+u_{52} \alpha\right)+ \\
& \left.\left.+b_{33} \sin \left(\alpha_{0}+\alpha\right) b_{23} \sin \left(\beta_{0}+u_{52} \alpha\right)\right]\right\}^{1 / 2},
\end{aligned}
$$

where

$$
\beta=u_{52} \alpha
$$

Here $\alpha$ is a variable value.

Analysis of expression (12) shows that distance $\ell$ depends on $\ell_{1}, \ell_{2}, \ell_{5}, \alpha_{0}, \beta_{0}, u_{52}, \alpha$ and directing cosines.

FROM triangle BCD taking into account the given maximal $\left(\gamma_{\max }\right)$ and minimal $\left(\gamma_{\min }\right)$ values of the angle of transmission can written:

$$
\begin{aligned}
& \ell_{3}^{2}+\ell_{4}^{2}-2 \ell_{3} \ell_{4} \cos \gamma_{\max }=\ell_{\text {max }}^{2} \\
& \ell_{3}^{2}+\ell_{4}^{2}-2 \ell_{3} \ell_{4} \cos \gamma_{\min }=\ell_{\text {min }}^{2}
\end{aligned}
$$

where $\ell_{\max }$ and $\ell_{\min }$ - are the extreme values of variable distance $\ell$.

It is known that if derivative $d \ell / d \alpha$ exists, then function $\ell$ will have maximum or minimum only if

$$
\frac{d \ell}{d \alpha}=0 .
$$

If second derivative $d^{2} \ell / d \alpha^{2}$ exists, then function $\ell$ will have maximum when

$$
\frac{d \ell}{d \alpha}=0, \quad \frac{d^{2} \ell}{d \alpha^{2}}<0
$$

and minimum at

$$
\frac{d \ell}{d \alpha}=0, \quad \frac{d^{2} \ell}{d \alpha^{2}}>0
$$

Accordingly we can write

$$
\begin{gathered}
\frac{d \ell}{d \alpha}=\frac{k^{\prime}}{2 \sqrt{k}} ; \\
\frac{d^{2} \ell}{d \alpha^{2}}=\frac{2 k k^{\prime \prime}-\left(k^{\prime}\right)^{2}}{4 \sqrt{k}},
\end{gathered}
$$

where

$$
\begin{aligned}
k= & \ell_{1}^{2}+\ell_{2}^{2}+\ell_{5}^{2}+2 \ell_{1} \ell_{2}\left[\cos \left(\alpha_{0}+\alpha\right) \cdot\right. \\
& \cdot\left(b_{12} \cos \xi+b_{12} \sin \xi\right)+\sin \left(\alpha_{0}+\alpha\right) \cdot \\
& \left.\cdot\left(b_{13} \cos \xi+b_{23} \sin \xi\right)\right]- \\
& -2 \ell_{1} \ell_{5} \sin \xi \cos \left(\beta_{0}+u_{52} \alpha\right)- \\
& -2 \ell_{2} \ell_{5}\left[b_{22} \cos \left(\alpha_{0}+\alpha\right) \cos \left(\beta_{0}+u_{52} \alpha\right)+\right. \\
& +b_{23} \sin \left(\alpha_{0}+\alpha\right) \cos \left(\beta_{0}+u_{52} \alpha\right)+ \\
& \left.+b_{33} \sin \left(\alpha_{0}+\alpha\right) \sin \left(\beta_{0}+u_{52} \alpha\right)\right] ; \\
k^{\prime}= & 2 \ell_{1} \ell_{2}\left[\cos \left(\alpha_{0}+\alpha\right)\left(b_{13} \cos \xi+b_{23} \sin \xi\right)-\right. \\
& \left.-\sin \left(\alpha_{0}+\alpha\right)\left(b_{12} \cos \xi+b_{22} \sin \xi\right)\right]+ \\
& +2 \ell_{1} \ell_{5}\left(u_{52}^{\prime} \alpha+u_{52}\right) \sin \xi \sin \left(\beta_{0}+u_{52} \alpha\right)- \\
& -2 \ell_{2} \ell_{5}\left[b _ { 2 3 } \left(\cos \left(\alpha_{0}+\alpha\right) \cos \left(\beta_{0}+u_{52} \alpha\right)-\right.\right. \\
& \left.-\left(u_{52}^{\prime} \alpha+u_{52}\right) \sin \left(\beta_{0}+u_{52} \alpha\right) \sin \left(\alpha_{0}+\alpha\right)\right)- \\
& -b_{22}\left(\sin \left(\alpha_{0}+\alpha\right) \cos \left(\beta_{0}+u_{52} \alpha\right)+\right. \\
& \left.+\left(u_{52}^{\prime} \alpha+u_{52}\right) \sin \left(\beta_{0}+u_{52} \alpha\right) \cos \left(\alpha_{0}+\alpha\right)\right)+ \\
& +b_{33}\left(\cos \left(\alpha_{0}+\alpha\right) \sin \left(\beta_{0}+u_{52} \alpha\right)+\right. \\
& \left.\left.+\left(u_{52}^{\prime} \alpha+u_{52}\right) \cos \left(\beta_{0}+u_{52} \alpha\right) \sin \left(\alpha_{0}+\alpha\right)\right)\right]
\end{aligned}
$$

The values k" will be found by differentiation of k'. In turn

$$
u_{52}^{\prime}=\frac{\beta^{\prime} \alpha-\beta}{\alpha^{2}} .
$$

For determining of such value of $\alpha$ that gives the extreme values of distance $\ell$ it is necessary that condition (14) be met. From the relations (17) we will have

$$
\begin{aligned}
& 2 \ell_{5} \cos u_{52} \alpha\left\{\ell_{1}\left(u_{52}^{\prime} \alpha+u_{52}\right) \sin \xi \sin \beta_{0}+\right. \\
& +\ell_{2} \sin \left(\alpha_{0}+\alpha\right)\left[b_{22} \cos \beta_{0}+\right. \\
& +b_{23}\left(u_{52}^{\prime} \alpha+u_{52}\right) \sin \beta_{0}- \\
& \left.-b_{33}\left(u_{52}^{\prime} \alpha+u_{52}\right) \cos \beta_{0}\right]+ \\
& +\ell_{2} \cos \left(\alpha_{0}+\alpha\right)\left[b_{22}\left(u_{52}^{\prime} \alpha+u_{52}\right) \sin \beta_{0^{-}}\right. \\
& \left.\left.-b_{23} \cos \beta_{0}-b_{33} \sin \beta_{0}\right]\right\}+ \\
& +2 \ell_{5} \sin u_{52} \alpha\left\{\ell_{1}\left(u_{52}^{\prime} \alpha+u_{52}\right) \sin \xi \sin \beta_{0}+\right. \\
& +\ell_{2} \sin \left(\alpha_{0}+\alpha\right)\left[b_{23} \cos \beta_{0}\left(u_{52}^{\prime} \alpha+u_{52}\right)-\right. \\
& \left.-b_{22} \sin \beta_{0}+b_{33}\left(u_{52}^{\prime} \alpha+u_{52}\right) \sin \beta_{0}\right]+ \\
& +\ell_{2}\left(\alpha_{0}+\alpha\right)\left[b_{22}\left(u_{52}^{\prime} \alpha+u_{52}\right) \cos \beta_{0}-\right.
\end{aligned}
$$




$$
\begin{aligned}
& \left.\left.-b_{23} \sin \beta_{0}-b_{33} \cos \beta_{0}\right]\right\}- \\
& -2 \ell_{1} \ell_{2}\left[\sin \left(\alpha_{0}+\alpha\right)\left(b_{12} \cos \xi+b_{22} \sin \xi\right)+\right. \\
& \left.+\cos \left(\alpha_{0}+\alpha\right)\left(b_{13} \cos \xi+b_{23} \sin \xi\right)\right]=0 .
\end{aligned}
$$

On the base of known formulae of the Moivre $\cos u_{52} \alpha$ and $\sin u_{52} \alpha$ included in the equation (20) can be presented as

$$
\begin{aligned}
\cos u_{52} \alpha= & \cos ^{u_{52}} \alpha-C_{u_{52}}^{2} \cos ^{u_{52}-2} \alpha \sin ^{2} \alpha+ \\
& +C_{u_{52}}^{4} \cos ^{u_{52}-4} \alpha \sin ^{4} \alpha-\cdots \\
\sin u_{52} \alpha= & u_{52} \cos ^{u_{52}-1} \alpha \sin \alpha- \\
& -C_{u_{52}}^{2} \cos ^{u_{52}-3} \alpha \sin ^{3} \alpha+ \\
& +C_{u_{52}}^{5} \cos ^{u_{52}-5} \alpha \sin ^{5} \alpha-\cdots
\end{aligned}
$$

Taking into account values (21) and (22) equation (20) will take a form:

$$
a_{1} \pm a_{2}-a_{3}=0
$$

where

$$
\begin{aligned}
a_{1}= & 2 \ell_{5}\left(\cos ^{u_{52}} \alpha-C_{u_{52}}^{2} \cos ^{u_{52}-2} \alpha \sin ^{2} \alpha+\right. \\
& \left.+C_{u_{52}}^{4} \cos ^{u_{52}-4} \alpha \sin ^{4} \alpha-\cdots\right) \cdot \\
& \cdot\left\{\ell_{1}\left(u_{52}^{\prime} \alpha+u_{52}\right) \sin \xi \sin \beta_{0}+\right. \\
& +\ell_{2} \sin \left(\alpha_{0}+\alpha\right)\left[b_{22} \cos \beta_{0}+\right. \\
& \left.+b_{23}\left(u_{52}^{\prime} \alpha+u_{52}\right) \sin \beta_{0}-b_{33}\left(u_{52}^{\prime} \alpha+u_{52}\right) \cos \beta_{0}\right]+ \\
& +\ell_{2} \cos \left(\alpha_{0}+\alpha\right)\left[b_{22}\left(u_{52}^{\prime} \alpha+u_{52}\right) \sin \beta_{0}-\right. \\
& \left.\left.-b_{23} \cos \beta_{0}-b_{33} \sin \beta_{0}\right]\right\} \\
a_{2}= & 2 \ell_{5}\left(u_{52} \cos ^{u_{52}-1} \alpha \sin \alpha-\right. \\
& -C_{u_{52}}^{2} \cos { }_{52}^{-3} \alpha \sin { }^{3} \alpha+ \\
& \left.+C_{u_{52}}^{2} \cos { }_{52}^{-5} \alpha \sin { }^{5} \alpha-\cdots\right) \\
& \cdot\left\{\ell_{1}\left(u_{52}^{\prime} \alpha+u_{52}\right) \sin \xi \sin \beta_{0}+\ell_{2} \sin \left(\alpha_{0}+\alpha\right) \cdot\right. \\
& \cdot\left[b_{23} \cos \beta_{0}\left(u_{52}^{\prime} \alpha+u_{52}\right)-b_{22} \sin \beta_{0}+\right. \\
& \left.+b_{33}\left(u_{52}^{\prime} \alpha+u_{52}\right) \cos \beta_{0}\right]+\ell_{2} \cos \left(\alpha_{0}+\alpha\right) \cdot \\
& \cdot\left[b_{22}\left(u_{52}^{\prime} \alpha+u_{52}\right) \cos \beta_{0}-\right. \\
& \left.\left.-b_{23} \sin \beta_{0}-b_{33} \cos \beta_{0}\right]\right\} \\
a_{3}= & 2 \ell_{1} \ell_{2}\left[\sin \left(\alpha_{0}+\alpha\right)\left(b_{12} \cos \xi+b_{22} \sin \xi\right)+\right. \\
& \left.+\cos \left(\alpha_{0}+\alpha\right)\left(b_{13} \cos \xi+b_{23} \sin \xi\right)\right] . \\
&
\end{aligned}
$$

Binomial coefficients in turn will have forms

$$
C_{u_{52}}^{2}=\frac{u_{52}\left(u_{52}-1\right)}{2 !} ;
$$

$$
\begin{aligned}
& C_{u_{52}}^{3}=\frac{u_{52}\left(u_{52}-1\right)\left(u_{52}-2\right)}{3 !} \\
& C_{u_{52}}^{4}=\frac{u_{52}\left(u_{52}-1\right)\left(u_{52}-2\right)\left(u_{52}-3\right)}{4 !} \\
& C_{u_{52}}^{5}=\frac{u_{52}\left(u_{52}-1\right)\left(u_{52}-2\right)\left(u_{52}-3\right)\left(u_{52}-4\right)}{5 !}
\end{aligned}
$$

and so on

$$
C_{u_{52}}^{n}=\frac{u_{52}\left(u_{52}-1\right)\left(u_{52}-2\right)\left(u_{52}-3\right) \cdots\left(u_{52}-(n-1)\right)}{n !} .
$$

In equations $(23)$ a sign $(+)$ is taken when gear ratios $u_{52}$ is a positive number, and sign (-) - when $u_{52}$ is a negative number.

The series of binomial coefficients is finite when $u_{52}$ is a positive or negative integer, and infinite when $u_{52}$ is a positive or negative fractional number.

By solution of equations (23) are determined the values $\alpha_{i}\left(\alpha_{1}, \alpha_{2}, \ldots, \alpha_{n}\right)$, with the help of which we determine extreme values $\ell_{\max }$ and $\ell_{\min }$ of distance $\ell$ accordingly to formula (12).

In practice are mainly encountered cases when the input links are moving with constant angular velocity $\left(u_{52}=\right.$ const). In such case expressions (17), (18) and equation (23) will be significantly simplified.

Thus knowing values $\ell_{\max }$ and $\ell_{\min }$, and transforming the system $(13)$ we obtain a value of coupler $4\left(\mathrm{DC}=\ell_{4}\right)$ length:

$$
\ell_{4}= \pm \sqrt{\frac{A_{2} \pm \sqrt{A_{2}^{2}-4 A_{1} A_{3}}}{2 A_{1}}}
$$

where

$$
\begin{aligned}
A_{1}= & 4\left(\cos \gamma_{\min }-\cos \gamma_{\max }\right)^{2} ; \\
A_{2}= & 4\left[\ell_{\text {max }}^{2}\left(\cos \gamma_{\min }-\cos \gamma_{\max }\right)^{2}+\right. \\
& \left.+\cos \gamma_{\max }\left(\ell_{\max }^{2}-\ell_{\min }^{2}\right)-\left(\cos \gamma_{\min }-\cos \gamma_{\max }\right)\right] ; \\
A_{3}= & \left(\ell_{\text {max }}^{2}-\ell_{\min }^{2}\right)^{2} .
\end{aligned}
$$

Accordingly a length of coupler $3\left(\mathrm{BC}=\ell_{3}\right)$ will be determined from system (13):

$$
\ell_{3}=\frac{\ell_{\max }^{2}-\ell_{\min }^{2}}{2 \ell_{4}\left(\cos \gamma_{\min }-\cos \gamma_{\max }\right)}
$$

\subsection{Determination of Limits of Angles of Transmission \\ Depending on Dimensions of Links of the Spatial Five-Bar Mechanism}

At solution of this problem a condition is envisaged according to which an angle of transmission at any position of the mechanism must not be less that certain given minimum value ensuring normal functioning of the mechanism.

Selection of allowable angles of transmission is determined by dynamical conditions of the mechanism operation and in the first place by absence of seizure of 
output links.

Considering a polygon of links $\mathrm{ABCDE}$ and triangle $\mathrm{BCD}$ (Fig. 1) for distance $\ell$ we can write:

$$
\begin{aligned}
\ell^{2}= & \ell_{1}^{2}+\ell_{2}^{2}+\ell_{5}^{2}+2 \ell_{1} \ell_{2}\left[\cos \left(\alpha_{0}+\alpha\right) \cdot\right. \\
& \cdot\left(b_{12} \cos \xi+b_{22} \sin \xi\right)+ \\
& \left.+\sin \left(\alpha_{0}+\alpha\right)\left(b_{31} \cos \xi+b_{23} \sin \xi\right)\right]- \\
& -2 \ell_{1} \ell_{5} \sin \xi \cos \left(\beta_{0}+u_{52} \alpha\right)- \\
& -2 \ell_{2} \ell_{5}\left[b_{22} \cos \left(\alpha_{0}+\alpha\right) \cos \left(\beta_{0}+u_{52} \alpha\right)+\right. \\
& +b_{23} \sin \left(\alpha_{0}+\alpha\right) \cos \left(\beta_{0}+u_{52} \alpha\right)+ \\
& \left.+b_{33} \sin \left(\alpha_{0}+\alpha\right) \sin \left(\beta_{0}+u_{52} \alpha\right)\right]= \\
& =\ell_{3}^{2}+\ell_{4}^{2}-2 \ell_{3} \ell_{4} \cos \gamma .
\end{aligned}
$$

From expressions (26) can be determined the angle of transmission $\gamma$ :

$$
\begin{aligned}
\cos \gamma= & \left(2 \ell_{3} \ell_{4}\right)^{-1}\left\{\ell_{3}^{2}+\ell_{4}^{2}-\ell_{1}^{2}-\ell_{2}^{2}-\ell_{5}^{2}-2 \ell_{1} \ell_{2}\right. \\
& {\left[\cos \left(\alpha_{0}+\alpha\right)\left(b_{12} \cos \xi+b_{22} \sin \xi\right)+\right.} \\
& \left.+\sin \left(\alpha_{0}+\alpha\right)\left(b_{31} \cos \xi+b_{23} \sin \xi\right)\right]+ \\
& +2 \ell_{1} \ell_{5} \sin \xi \cos \left(\beta_{0}+u_{52} \alpha\right)+ \\
& +2 \ell_{2} \ell_{5}\left[b_{22} \cos \left(\alpha_{0}+\alpha\right) \cos \left(\beta_{0}+u_{52} \alpha\right)+\right. \\
& +b_{23} \sin \left(\alpha_{0}+\alpha\right) \cos \left(\beta_{0}+u_{52} \alpha\right) \\
& \left.+b_{33} \sin \left(\alpha_{0}+\alpha\right) \sin \left(\beta_{0}+u_{52} \alpha\right)\right\} .
\end{aligned}
$$

If $0<\gamma<\pi$, then the extreme values of angle $\gamma$ will be determined from formula (27):

$$
\begin{aligned}
& \cos \gamma_{\max }=\frac{\ell_{3}^{2}+\ell_{4}^{2}-\ell_{\max }^{2}}{2 \ell_{3} \ell_{4}}, \\
& \cos \gamma_{\min }=\frac{\ell_{3}^{2}+\ell_{4}^{2}-\ell_{\min }^{2}}{2 \ell_{3} \ell_{4}} .
\end{aligned}
$$

An optimal value $\gamma=\pi / 2$ will be obtained at value of variable angle $\alpha$ determined from equation

$$
\begin{aligned}
& \ell_{3}^{2}+\ell_{4}^{2}-\ell_{1}^{2}-\ell_{2}^{2}-\ell_{5}^{2}- \\
& -2 \ell_{1} \ell_{2}\left[\cos \left(\alpha_{0}+\alpha\right)\left(b_{12} \cos \xi+b_{12} \sin \xi\right)+\right. \\
& \left.+\sin \left(\alpha_{0}+\alpha\right)\left(b_{13} \cos \xi+b_{23} \sin \xi\right)\right]+ \\
& +2 \ell_{1} \ell_{5} \sin \xi \cos \left(\beta_{0}+u_{52} \alpha\right)+ \\
& +2 \ell_{2} \ell_{5}\left[b_{22} \cos \left(\alpha_{0}+\alpha\right) \cos \left(\beta_{0}+u_{52} \alpha\right)+\right. \\
& +b_{23} \sin \left(\alpha_{0}+\alpha\right) \cos \left(\beta_{0}+u_{52} \alpha\right)+ \\
& \left.+b_{33} \sin \left(\alpha_{0}+\alpha\right) \sin \left(\beta_{0}+u_{52} \alpha\right)\right]=0 .
\end{aligned}
$$

The obtained results allow to determine relations between dimensions of links of the spatial five-bar mechanism for which optimal value of the angle of transmission will be arithmetical mean of its extreme values, i.e.

$$
\gamma=\frac{\pi}{2}=\frac{\gamma_{\max }-\gamma_{\min }}{2}
$$

Suppose

$$
\gamma_{\max }=\frac{\pi}{2}+\delta \text { and } \gamma_{\min }=\frac{\pi}{2}-\delta
$$

where $\delta$ is a certain angle.

Substituting of these values in (28) and (29) we will have:

$$
\begin{aligned}
& -2 \ell_{3} \ell_{4} \sin \delta=\ell_{3}^{2}+\ell_{4}^{2}-\ell_{\text {max }}^{2} \\
& 2 \ell_{3} \ell_{4} \sin \delta=\ell_{3}^{2}+\ell_{4}^{2}-\ell_{\text {min }}^{2} .
\end{aligned}
$$

Excluding $\delta$ we will obtain

$$
2\left(\ell_{3}^{2}+\ell_{4}^{2}\right)=\ell_{\max }^{2}+\ell_{\min }^{2} .
$$

Thus if dimensions of links of the spatial five-bar mechanism satisfy condition (34), then deviations of the angle of transmission $\gamma$ from optimal value $\gamma=\pi / 2$ will be identical in both sides.

\subsection{Conditions of Existence of Two Cranks in Spatial Five-Bar Mechanisms}

At solution of numerous problems of synthesis of spatial mechanisms with two degrees of freedom it is very important to select relations between lengths of the links allowing one or two links to realize full or partial turning around chosen points.

It is known that condition of existence of cranks in planar and spherical four-bar mechanisms was first formulated by $F$. Grasgof. The similar problems were studied by N.G. Bruevich, V.V. Dobrovolski, F. Freidenstein, F. Duditsa and others.

For spatial four-bar mechanisms conditions of existence of one of two cranks was first formulated by D.S. Tavkhelidze.

By numerous researches into various hinged mechanisms by N.S. Davitashvili were formulated theorems of existience of cranks in spherical five-bar, six-bar and seven-bar mechanisms.

By studies of structure and kinematics of five-bar mechanisms with two degrees of freedom is ascertained that conditions of turning of input links are significantly influenced by dimensions of frame 1 and links 2 and 5 (Fig. 1), gear ratio $\mathrm{u}_{52}$ between input links considering their directions of movement, initial positions of input links specified by angles $\alpha_{0}$ and $\beta_{0}$ and duration of the mechanism movement cycle.

For spatial five-bar mechanism (Fig. 1) we have obtained formula (12) for calculation of variable distance $\ell$ between movable hinges $B$ and $D$, whose extreme values $\ell_{\max }$ and $\ell_{\text {min }}$ are found on the base of satisfaction of conditions (15), (16).

The found extreme values $\ell_{\max }$ and $\ell_{\min }$ of distance $\ell$ allow us to write inequalities defining conditions of existence of cranks 2 and 5according to the kinematical scheme of spatial five-bar mechanism:

$$
\begin{aligned}
& \ell_{\max } \leq \ell_{3}+\ell_{4} \\
& \ell_{\min } \geq\left|\ell_{3}-\ell_{4}\right| .
\end{aligned}
$$

Thus we can formulate a theorem of existence of two cranks 
in spatial five-bar hinged mechanisms as follows.

In spatial five-bar hinged mechanisms two links, adjoining to the frame, will be cranks if and only if at movement of the mechanism maximum distance between the hinges created by connection of input links and corresponding couplers is less or equal to the sum of lengths of couplers and minimum distance is more or equal to the difference of these lengths.

If the assume that $\alpha 0=\beta 0=0$ and $\ell 5=0$ the spatial five-bar mechanism ABSDE (Fig. 1) will be converted into spatial four-bar mechanism ABCD (Fig. 2).

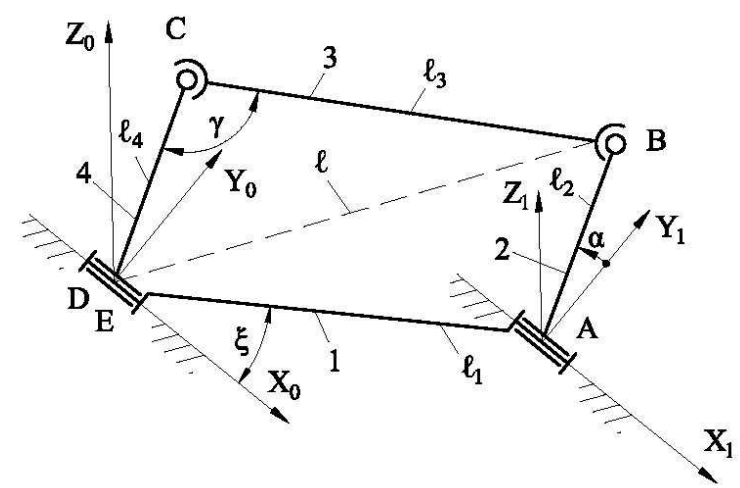

Figure 2. On the conditions of existence of cranks in a spatial four-bar mechanism.

In this case variable distance $\ell$ according to expression (12) will be:

$$
\begin{aligned}
\ell= & \left\{\ell_{1}^{2}+\ell_{2}^{2}+2 \ell_{1} \ell_{2}\left[\cos \alpha\left(b_{12} \cos \xi+b_{22} \sin \xi\right)+\right.\right. \\
& \left.\left.+\sin \alpha\left(b_{31} \cos \xi+b_{23} \sin \xi\right)\right]\right\}^{1 / 2}
\end{aligned}
$$

The equation for finding $\alpha$ will be written as:

$$
\begin{gathered}
2 \ell_{1} \ell_{2}\left[\cos \alpha\left(b_{13} \cos \xi+b_{23} \sin \xi\right)-\right. \\
\left.-\sin \alpha\left(b_{12} \cos \xi+b_{22} \sin \xi\right)\right]=0 .
\end{gathered}
$$

Accordingly value of $\alpha$ will be found by formula

$$
\cos \alpha_{(1,2)}= \pm \frac{b_{1}}{\sqrt{b_{1}^{2}+b_{2}^{2}}}
$$

where

$$
\begin{aligned}
& b_{1}=b_{12} \cos \xi+b_{22} \sin \xi \\
& b_{2}=b_{13} \cos \xi+b_{23} \sin \xi
\end{aligned}
$$

Taking into account the values of $\alpha_{1}$ and $\alpha_{2}$ from expression (36) we will have:

$$
\begin{aligned}
\ell_{\text {max }}= & {\left[\ell_{1}^{2}+\ell_{2}^{2}+2 \ell_{1} \ell_{2}\left(b_{12} \cos \alpha_{1}+\right.\right.} \\
& \left.+b_{22} \sin \alpha_{1}\right]^{1 / 2} \\
\ell_{\text {min }}= & {\left[\ell_{1}^{2}+\ell_{2}^{2}+2 \ell_{1} \ell_{2}\left(b_{12} \cos \alpha_{2}+\right.\right.} \\
& \left.+b_{22} \sin \alpha_{2}\right]^{1 / 2} .
\end{aligned}
$$

Accordingly to expressions (35) conditions of existence of cranks in the spatial four-bar mechanism will be:

$$
\begin{aligned}
& {\left[\ell_{1}^{2}+\ell_{2}^{2}+2 \ell_{1} \ell_{2}\left(b_{1} \cos \alpha_{1}+b_{2} \sin \alpha_{1}\right)\right]^{1 / 2} \leq} \\
& \leq \ell_{3}+\ell_{4} \\
& {\left[\ell_{1}^{2}+\ell_{2}^{2}+2 \ell_{1} \ell_{2}\left(b_{1} \cos \alpha_{2}+b_{2} \sin \alpha_{2}\right)\right]^{1 / 2} \geq} \\
& \geq\left|\ell_{3}-\ell_{4}\right| .
\end{aligned}
$$

\section{Conclusion}

By accomplished researches into five-bar mechanisms with two degrees of freedom are marked out some problems of synthesis of the mentioned mechanisms.

For the purpose of ensuring operation of the mechanism without seizure with maximum possible efficiency are determined dimensions of the links firstly considering a given angle of transmission and then depending on the dimensions of links of the spatial five-bar mechanism are calculated limits of variation of the angle of transmission. A theorem of existence of two cranks in spatial five-bar hinged mechanisms is formulated. The obtained results are suitable for designing new mechanisms and solution of practical problems.

\section{References}

[1] G. Eason, B. Noble, and I. N. Sneddon, "On certain integrals of Lipschitz-Hankel type involving products of Bessel functions," Phil. Trans. Roy. Soc. London, vol. A247, pp. 529-551, April 1955. (references)

[2] J. Clerk Maxwell, A Treatise on Electricity and Magnetism, 3rd ed., vol. 2. Oxford: Clarendon, 1892, pp.68-73.

[3] A. G. Ovakimov, "Task on positions of spatial mechanisms with several degree of freedom and its solution by closed vector contour method" in Mechanics of Machines, issue 29-23, Moscow: Nauka, 1971, pp. 61-75. (In Russian).

[4] J. Duffy, H.Y. Habib-Olahi, “A displacement analysis of spatial five-link BR-2C mechanism . 1. On the clousers of the RCRCR mechanism” in Journal of Mechanics, vol. 5, № 3, 1971, pp. 289-301.

[5] J. Duffy, "A derivation of dual displacement equations for five-size-and seven-link spatial mechanism using spherical trigonometry. 3. Deviation of dual equations for seven-link spatial mechanism s" Rev. roum. sci. techn. Scr. mech. appl. vol. 17, 1972, № 2, pp. 291-317.

[6] N.S. Davitashvili, "Influence of parameters of five-bar hinged mechanism on trajectory of coupler's points," Transactions of Academy of Sciences of GSSR, vol. 86, 1977, № 2, pp. 412-420, (In Russian).

[7] N.S. Davitashvili, "Issues of kinematics and precision of five-bar hinged mechanism" Fifth World congress on Theory of Machines and Mechanisms, vol. 1, 1979, Canada, Motreal, July 8-13, pp. 557-561.

[8] N.S. Davitashvili, "Fundamentals of the theory of synthesis, analysis and precision of linkage-lever mechanisms", Tbilisi: Technical University, 1999. -388 p. (In Russian).

[9] N.S. Davitashvili, "Theoretical fundamentals of error and precision of hinged mechanisms with two degrees of freedom", Tbilisi: Metsniereba, 2000. -286 p. (In Russian). 
[10] N.S. Davitashvili, "Dynamic investigation of plane five-link hinged mechanism with two degrees of freedom ",Tbilisi: Georgian Committee of IFToMM, 2006. - 196 p. (In Russian).

[11] N. Davitashvili, O. Gelashvili, "Synthesis of a spatial five-link mechanism with two degrees of freedom according to the given laws of Motion, in Proceedings of EUCOMES 08, the second European Congress on Mechanism Science, Springer, pp. 159-166. 